\title{
Adaptive Simple Pendulum Swing-up Controller based on the Closed-Loop Fundamental Dynamics*
}

\author{
Philine Donner $^{1,2}$, Franz Christange ${ }^{1}$ and Martin Buss ${ }^{1,2}$
}

\begin{abstract}
An adaptive energy-based swing-up controller for simple pendulums is presented. A state transformation from cartesian to polar phase space followed by approximation steps leads to the fundamental dynamics of the controlled simple pendulum. Based on the fundamental dynamics, the unknown natural frequency is estimated and a control gain is adjusted such that the system energy follows desired reference dynamics. We prove convergence of the natural frequency estimate to the true value as well as convergence of the system energy to the desired energy level under the fundamental dynamics assumption. The implications of the fundamental dynamics approximation are evaluated in simulation. Noise affected angle measurements simulate a realistic inverted pendulum experiment. The successful swing-up into the highly nonlinear regimes of the simple pendulum support the applicability of the fundamental dynamics.
\end{abstract}

\section{INTRODUCTION}

Simple pendulums have been thoroughly studied. Their nonlinear nature has turned them into an extensively used test bed for linear as well as nonlinear control approaches. Next to serving as a control theoretic test bed, simple pendulums have been successfully used to approximate complex mechanisms. Examples are robotic walking [1], robotic brachiation [2] or the manipulation of pendulum-like objects [3]. All examples mentioned share the need for energy control. In order to reach stable limit cycles when the environment, e.g. the ground, changes or the manipulated objects are unknown, adaptive control approaches are needed. This motivates the design of an adaptive energy-based swing-up controller for simple pendulums of unknown dimensions in this paper.

In the literature, different swing-up controllers are found for different types of pendulums. The cart driven simple pendulum [4], the acrobot [5], the pendubot [6] and the furuta pendulum [7] are pendulum variants with one underactuated degree of freedom. Our work focuses on cart driven simple pendulums, a pendulum attached to a horizontally moving pivot (see Fig. 1). Most swing-up controllers are designed based on energy considerations. In [4], energybased controllers with minimal swing-up time for cart driven simple pendulums are investigated with respect to the ratio of maximum acceleration to gravity. However, only the acceleration is considered. Examples for approaches that take

\footnotetext{
*This work was supported in part within the ERC Advanced Grant SHRINE Agreement No. 267877 (www.shrine-project.eu) and in part by the Technische Universität München - Institute for Advanced Study (www.tumias.de), funded by the German Excellence Initiative

${ }^{1}$ Chair of Automatic Control Engineering, Technische Universität München, Theresienstr. 90, 80333 München, Germany, \{philine.donner, franz.christange, mb\}etum.de

${ }^{2}$ TUM Institute for Advanced Study, Technische Universität München, Lichtenbergstrasse 2a, 85748 Garching, Germany
}

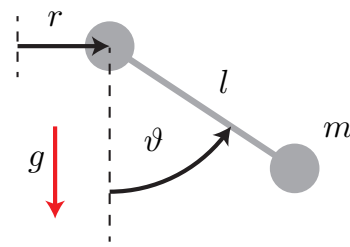

Fig. 1. Simple pendulum under the influence of gravity $g$

into account restricted pivot travel are e.g. [8], [9], [10]. Lozano et al. present a passivity based design of a swingup controller and show asymptotic stability of the inverted pendulum at zero cart displacement [9]. The energy-based controller by Yoshida injects energy into the pendulum by excitation at its natural frequency, while obeying a restricted pivot travel by means of a sinusoidal reference input with low-pass properties [10].

Application of swing motion in robotics is motivated by an increased workspace [11] as well as extended locomotion [2] and manipulation capabilities [3]. Ape-like brachiation is achieved in [2] by controlling a two-link robot to follow certain target dynamics. For the swing-up problem the target dynamics mimic a negatively damped simple pendulum. Human-robot cooperative swinging of complex pendulumlike objects is discussed in [3]. The pendulum-like object is projected onto a simple pendulum. The energy of the virtual simple pendulum is controlled based on the swing-up controller presented in [10].

All the afore mentioned works are model-based. Only few works on adaptive swing-up controllers exist. A fuzzy controller with an adaptive mechanism to online adjust the controller parameters is presented in [12]. The model-based swing-up controller of [10] is combined with a reinforcement learning algorithm in the context of human-robot object swinging in [13]. The major drawback of the reinforcement learning approach is the high number of tuning parameters.

The adaptive simple-pendulum swing-up controller discussed in this paper builds upon the same model-based controller [10] as the reinforcement learning approach [13]. The main contribution of this work are the fundamental dynamics which approximate the simple pendulum dynamics. The fundamental dynamics enable the design of a simple adaptation mechanism for the natural frequency of the pendulum and the adjustment of a control gain such that the system energy follows desired reference dynamics. In contrast to the reinforcement learning approach in [13], only two design parameters are introduced by the adaptive control; namely the gain of the reference dynamics and a time constant that 
defines the speed of the natural frequency adaptation.

The remainder of this paper is organized as follows. Section II recapitulates important simple pendulum basics, describes the underlying model-based swing-up controller and finishes with the problem statement for the adaptive swing-up controller. The fundamental dynamics are derived in Section III, based on which the adaptive controller including stability analyses is developed in Section IV. Section V investigates the interaction of the adaptive controller with a simple pendulum in simulation. We draw our conclusions in Section VI.

\section{PROBLEM FORMULATION}

In this section we first recapitulate important simple pendulum basics (Section II-A). This is followed by a description of the model-based controller by [10] in Section II-B. Based on the knowledge of the underlying model-based controller, the problem statement for the adaptive swing-up controller is formulated in Section II-C.

\section{A. Simple pendulum preliminaries}

Figure 1 shows a simple pendulum, which consists of a point mass $m$ attached through a massless rod of length $l$ to a pivot located at $r$. The pendulum states $\boldsymbol{x}_{\mathrm{c}}=\left(\begin{array}{ll}\vartheta & \dot{\vartheta}\end{array}\right)^{T}$ are influenced by horizontal acceleration of the pivot $u=\ddot{r}$. The state space representation of a lossless simple pendulum is

$$
\dot{\boldsymbol{x}}_{\mathrm{c}}=\left(\begin{array}{c}
\dot{\vartheta} \\
-\omega_{0}^{2} \sin \vartheta
\end{array}\right)+\left(\begin{array}{c}
0 \\
-\frac{1}{g} \omega_{0}^{2} \cos \vartheta
\end{array}\right) \ddot{r},
$$

with gravity $g$ and the small angle approximation of the natural frequency $\omega_{0}=\sqrt{\frac{g}{l}}$. The simple pendulum is controllable via $u=\ddot{r}$ everywhere except for deflection angles $\vartheta= \pm \pi / 2$.

We define the energy $E_{\vartheta}$ of the $\vartheta$-oscillation to be the energy of the simple pendulum for zero pivot velocity $\dot{r}=0$

$$
E_{\vartheta}:=\left.E\right|_{\dot{r}=0}=\frac{1}{2} m l^{2} \dot{\vartheta}^{2}+m g l(1-\cos \vartheta) .
$$

Differentiation of the energy $E_{\vartheta}$ with respect to time and insertion of the pendulum dynamics (1) leads to the energy flow $\dot{E}_{\vartheta}$ to the pendulum caused by the acceleration input $\ddot{r}$

$$
\dot{E}_{\vartheta}=-m l \dot{\vartheta} \cos \vartheta \ddot{r} .
$$

Consequently, energy cannot be injected when the pendulum is uncontrollable $(\vartheta= \pm \pi / 2)$ and at the turning points $(\dot{\vartheta}=0)$. Energy is most efficiently injected for maximum angular velocities $\dot{\vartheta}$, which occur when the pendulum passes the lower equilibrium point $\vartheta=0$.

The energy $E_{\vartheta}$ can equivalently be expressed as the amplitude $\vartheta_{E}$ of the $\vartheta$-oscillation. The amplitude $\vartheta_{E}$ equals the maximum deflection angle $\vartheta$ reached at the turning points

$$
\begin{aligned}
E_{\vartheta} & \stackrel{!}{=} m g l\left(1-\cos \vartheta_{E}\right) \\
\Leftrightarrow \vartheta_{E} & =\arccos \left(1-\frac{E_{\vartheta}}{m g l}\right) \\
& \stackrel{(2)}{=} \arccos \left(\cos \vartheta-\frac{l}{2 g} \dot{\vartheta}^{2}\right) .
\end{aligned}
$$

Note that $\vartheta_{E} \in[0, \pi]$, meaning that the energy of an inverted pendulum is the maximum representable energy through $\vartheta_{E}$, which suffices for our purposes. Throughout the rest of the paper, we use the amplitude $\vartheta_{E}$ to refer to the system energy.

The natural frequency of the nonlinear simple pendulum is not constant, but decreases with higher energy content $\omega\left(\vartheta_{E}\right)$. No analytic solution exists for the natural frequency, but it can be obtained numerically by the arithmeticgeometric mean $M\left\{1, \cos \frac{\vartheta_{E}}{2}\right\}$ [14]

$$
\begin{aligned}
\omega & =\omega_{0} M\left\{1, \cos \frac{\vartheta_{E}}{2}\right\} \\
\omega_{\mathrm{a}} & =\omega_{0} \frac{1+\cos \frac{\vartheta_{E}}{2}}{2} \\
\omega_{\mathrm{g}} & =\omega_{0} \sqrt{\cos \frac{\vartheta_{E}}{2}},
\end{aligned}
$$

where $\omega_{\mathrm{a}}$ and $\omega_{\mathrm{g}}$ are the arithmetic and geometric mean of $\left\{1, \cos \frac{\vartheta_{E}}{2}\right\}$ multiplied by the small angle approximation of the natural frequency $\omega_{0}$. The arithmetic and geometric mean are the result of the first iteration of the arithmeticgeometric mean $M\left\{1, \cos \frac{\vartheta_{E}}{2}\right\}$ calculation and already represent good approximations for $\omega\left(\vartheta_{E}\right)$ : The maximum error at $\vartheta_{E}=\frac{\pi}{2}$ is $0.74 \%$ for $\omega_{\mathrm{a}}$ and $0.76 \%$ for $\omega_{\mathrm{g}}$.

The nonlinearities of the simple pendulum are also visible in its phase space. The left hand side of Fig. 2 shows the phase portrait for two different constant energy levels $\vartheta_{E}=$ $\{0.5 \pi, 0.9 \pi\}$. The inscribed phase angle $\varphi$ is calculated as

$$
\varphi=\operatorname{atan} 2\left(-\frac{\dot{\vartheta}}{\Omega}, \vartheta\right),
$$

with the variable $\Omega$ serving as a normalization factor. On the right hand side of Fig. 2 the phase angle $\varphi$ is plotted over time for zero initial phase $\varphi(t=0)=0$. Normalization with the natural frequency $\Omega=\omega$ leads to a circle-like phase portrait for relatively low energy levels and consequently a linearly rising phase angle

$$
\varphi(t) \approx \omega t+\varphi(t=0) .
$$

The higher the energy content of the simple pendulum, the more apparent are the nonlinearities in the phase space as well as in $\varphi(t)$ (see Fig. 2). Normalization with the small angle approximation $\Omega=\omega_{0}$ leads to an increased deviation from a circle-like phase portrait, especially for high energy contents.

\section{B. Model based swing-up controller}

The adaptive control approach presented in this paper builds upon the model-based swing-up controller by Yoshida [10]. The following approximate control law captures the idea of the energy-based swing-up controller

$$
\ddot{r} \approx a \omega^{2} \sin \varphi,
$$

where $a$ is an amplitude factor, which we will later use to specify the amount and direction of energy flow to the pendulum. From the approximate relationship (7) we can infer that the term $\sin \varphi$ allows for a well-timed energy 

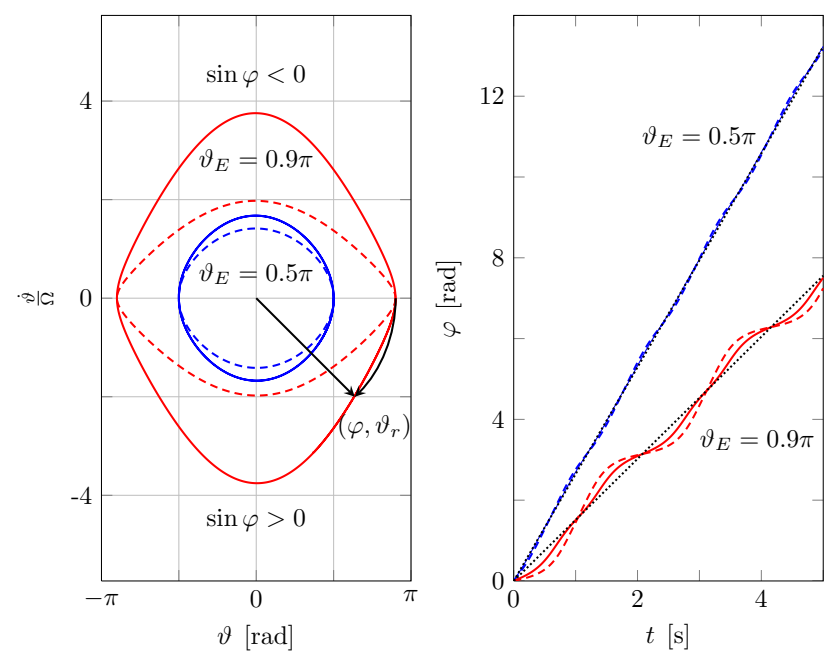

Fig. 2. Phase portrait of a lossless simple pendulum (left) and the resulting phase angle $\varphi$ over time (right) for two different energy levels $\vartheta_{E}$. Solid lines indicate normalization with $\Omega=\omega$, dashed lines $\Omega=\omega_{0}$.

injection, as it excites the pendulum close to its natural frequency $\omega$. In [10], the small angle approximation is used for normalization $\Omega=\omega_{0}$ within the phase angle $\varphi$ calculation (6).

We explain the influence of the amplitude factor $a$ in the following. According to (3), the energy flow is positive if $\operatorname{sgn}(\dot{\vartheta} \cos \vartheta)=-\operatorname{sgn}(\ddot{r})$. For angles $\vartheta=]-\frac{\pi}{2}, \frac{\pi}{2}[$, $\cos \vartheta>0$ and further $\operatorname{sgn}(\dot{\vartheta})=-\operatorname{sgn}(\sin \varphi)$ (see Fig. 2). Consequently, the approximate control law (8) with a positive amplitude factor $a>0$ leads to positive energy flow $\dot{E}_{\vartheta}>0$, whereas a negative amplitude factor $a<0$ leads to negative energy flow $\dot{E}_{\vartheta}<0$.

The amplitude factor $a$ is calculated based on a linear mapping from the difference of the current energy $E_{\vartheta}$ to the desired energy $E_{\vartheta}^{\mathrm{d}}$ saturated at $\pm \bar{a}$

$$
a=\left\{\begin{array}{cl}
\bar{a} \operatorname{sgn}\left(E_{\vartheta}^{\mathrm{d}}-E_{\vartheta}\right) & \text { if } k\left|E_{\vartheta}^{\mathrm{d}}-E_{\vartheta}\right| \geq \bar{a} \\
k\left(E_{\vartheta}^{\mathrm{d}}-E_{\vartheta}\right) & \text { else, }
\end{array}\right.
$$

with $k$ being a control gain which defines the slope of the linear mapping. The saturation allows to specify workspace boundaries. From (8) we can infer that the pivot trajectory is approximately $r \approx-a \sin \varphi$. Thus, the limits $\pm \bar{a}$ relate to the maximum pivot amplitude $r$.

For energies $\vartheta_{E}$ higher than $\frac{\pi}{2}$, periods of $\cos \vartheta<0$ occur which violate the sign condition. However, within these periods energy flow $\left|\dot{E}_{\vartheta}\right|$ is relatively low, allowing to use the same control law also for desired energies $\vartheta_{E}>\frac{\pi}{2}$ [10].

As explained in the previous section, the phase $\varphi$ is only approximately time linear. Consequently, the acceleration $\ddot{r}$ according to the approximate control law (8) does not only contain the desired oscillation of frequency $\omega$, but also higher order harmonics, which disturb the sign condition. In order to attenuate the higher harmonics and to be able to obey a restricted workspace, Yoshida defines a reference trajectory [10]

$$
r^{\mathrm{d}}=-\frac{a}{|G(j \Omega)|} \sin (\varphi-\angle G(j \Omega))
$$

which is filtered by the second-order low-pass filter

$$
G(s)=\frac{\ddot{r}}{r^{\mathrm{d}}}=\frac{s^{2}\left(\frac{\Omega}{c_{\mathrm{f}}}\right)^{2}}{s^{2}+2 \zeta \frac{\Omega}{c_{\mathrm{f}}} s+\left(\frac{\Omega}{c_{\mathrm{f}}}\right)^{2}},
$$

with design parameters $c_{\mathrm{f}}$ and $\zeta$. The acceleration results approximately in

$$
\begin{aligned}
\ddot{r} \simeq a \omega^{2} \frac{|G(j \omega)|}{|G(j \Omega)|} \sin (\varphi-\angle G(j \Omega) & +\angle G(j \omega)) \\
& \approx a \omega^{2} \sin (\varphi) .
\end{aligned}
$$

The sinusoidal shape of the commanded robot acceleration allows to compensate for the amplitude and phase shift $|G(j \omega)|$ and $\angle G(j \omega)$ caused by the filter (11) at the natural frequency $\omega$, by using the approximated amplitude and phase shift $|G(j \Omega)|$ and $\angle G(j \Omega)$ with $\Omega=\omega_{0}$ within the reference trajectory (10).

\section{Problem statement for the adaptive swing-up controller}

The energy-based swing-up controller by Yoshida requires model knowledge. The objective of our work is to design a swing-up controller that allows to control the energy of simple pendulums with unknown length $l$ and mass $m$. We are looking for a control law

$$
\begin{gathered}
u=\ddot{r}=f\left(\boldsymbol{x}_{\mathrm{c}}\right) \\
\text { s. t. }\left|\vartheta_{E}-\vartheta_{E \mathrm{~m}}\right| \leq \epsilon \\
\text { with } \quad \dot{\vartheta}_{E \mathrm{~m}}=K_{\mathrm{d}}\left(\vartheta_{E}^{\mathrm{d}}-\vartheta_{E \mathrm{~m}}\right),
\end{gathered}
$$

where $\epsilon$ defines bounds within which the system energy $\vartheta_{E}$ is required to follow the first order reference dynamics of $\vartheta_{E \mathrm{~m}}$. We assume state feedback $\boldsymbol{x}_{\mathrm{c}}=\left(\begin{array}{ll}\vartheta & \dot{\vartheta}\end{array}\right)^{T}$.

By the use of the amplitude $\vartheta_{E}$ instead of the energy $E_{\vartheta}$ as an energy equivalent (see (4)), the controller becomes independent of mass $m$. The small angle approximation of the natural frequency $\omega_{0}=\sqrt{\frac{g}{l}}$ is the only unknown model parameter that has to be adapted. ${ }^{1}$ We further want to adapt the control gain $k$ of (9) such that the system energy $\vartheta_{E}$ follows the reference dynamics of $\vartheta_{E \mathrm{~m}}$.

\section{FUNDAMENTAL DYNAMICS}

In this section we transform the system states from cartesian to polar coordinates. The resultant state space representation is simplified through approximations with Taylor polynomials and negligence of higher harmonics. The result are approximately decoupled system dynamics, based on which we design an adaptive controller in Section IV.

\section{A. Cartesian to polar state transformation}

The dynamics (1) are highly coupled with respect to the states $x_{\mathrm{c}}=\left(\begin{array}{ll}\vartheta & \dot{\vartheta}\end{array}\right)^{T}$. We add the index c to indicate that the angle $\vartheta$ and angular velocity $\dot{\vartheta}$ represent the cartesian coordinates in the phase space (see Fig. 2). A state transformation to the phase of the oscillation $\varphi$ and the energy

\footnotetext{
${ }^{1}$ Note that this can also be achieved for a simple pendulum with distributed mass through normalization [4].
} 
equivalent $\vartheta_{E}$ would ideally lead to decoupled states. This refers to the expectation that the energy of the system is independent of the phase of the oscillation. Insertion of (6) solved for $\dot{\vartheta}$ into (4) yields

$$
\cos \vartheta_{E}=\cos \vartheta-\frac{\Omega^{2}}{2 \omega_{0}^{2}} \tan ^{2} \varphi \vartheta^{2} .
$$

This relationship is, however, not analytically solvable for $\vartheta$. We circumvent this problem by using the radius of the phase space $\vartheta_{r}$ to approximate the system energy $\vartheta_{E}$

$$
\vartheta_{r}:=\sqrt{\vartheta^{2}+\left(\frac{\dot{\vartheta}}{\Omega}\right)^{2}} .
$$

Note that $\vartheta_{r}$ approximates the energy $\vartheta_{E}$, but is superimposed by higher order oscillations, which increase with higher energy content of the system $\vartheta_{r} \approx$ const $\forall E_{\vartheta}=$ const (see Fig. 2).

The phase $\varphi$ and the radius $\vartheta_{r}$ span the new phase space $\boldsymbol{x}_{\mathrm{p}}=\left(\begin{array}{ll}\varphi & \vartheta_{r}\end{array}\right)^{T}$, with $\mathrm{p}$ indicating polar coordinates. The cartesian states $\boldsymbol{x}_{\mathrm{c}}$ can be expressed as a function of the polar states $\boldsymbol{x}_{\mathrm{p}}$

$$
\begin{aligned}
& \vartheta=\vartheta_{r} \cos \varphi \\
& \dot{\vartheta}=-\vartheta_{r} \Omega \sin \varphi .
\end{aligned}
$$

We obtain the dynamical equations for the polar states $\boldsymbol{x}_{\mathrm{p}}$ in three steps: Differentiation of (6) and (15) with respect to time, insertion of the cartesian state dynamics (1) and substitution of the remaining cartesian states through polar states (16)

$$
\begin{aligned}
& \dot{\varphi}=\frac{\Omega \dot{\vartheta}^{2}-\Omega \vartheta \ddot{\vartheta}}{\Omega^{2} \vartheta^{2}+\dot{\vartheta}^{2}} \\
& \stackrel{(1)}{=} \frac{\Omega \dot{\vartheta}^{2}+\Omega \omega_{0}^{2} \vartheta \sin \vartheta+\frac{1}{g} \Omega \omega_{0}^{2} \vartheta \cos \vartheta \ddot{r}}{\Omega^{2} \vartheta^{2}+\dot{\vartheta}^{2}} \\
& \stackrel{(16)}{=} \Omega \sin ^{2} \varphi+\frac{\omega_{0}^{2}}{\Omega \vartheta_{r}} \cos \varphi \sin \left(\vartheta_{r} \cos \varphi\right) \\
& +\frac{\omega_{0}^{2}}{g \Omega \vartheta_{r}} \cos \varphi \cos \left(\vartheta_{r} \cos \varphi\right) \ddot{r}, \\
& \dot{\vartheta}_{r}=\frac{\Omega^{2} \vartheta \dot{\vartheta}+\dot{\vartheta} \ddot{\vartheta}}{\Omega \sqrt{\Omega^{2} \vartheta^{2}+\dot{\vartheta}^{2}}} \\
& \stackrel{(1)}{=} \frac{\Omega^{2} \vartheta \dot{\vartheta}-\omega_{0}^{2} \dot{\vartheta} \sin \vartheta-\frac{1}{g} \omega_{0}^{2} \dot{\vartheta} \cos \vartheta \ddot{r}}{\Omega \sqrt{\Omega^{2} \vartheta^{2}+\dot{\vartheta}^{2}}} \\
& \stackrel{(16)}{=}-\Omega \vartheta_{r} \sin \varphi \cos \varphi+\frac{\omega_{0}^{2}}{\Omega} \sin \varphi \sin \left(\vartheta_{r} \cos \varphi\right) \\
& +\frac{\omega_{0}^{2}}{g \Omega} \sin \varphi \cos \left(\vartheta_{r} \cos \varphi\right) \ddot{r} .
\end{aligned}
$$

The resultant state space representation is of the form

$$
\dot{\boldsymbol{x}}_{\mathrm{p}}=\boldsymbol{f}_{\mathrm{p}}\left(\boldsymbol{x}_{\mathrm{p}}\right)+\boldsymbol{g}_{\mathrm{p}}\left(\boldsymbol{x}_{\mathrm{p}}\right) \ddot{r}
$$

and still highly coupled.

\section{B. Extraction of the fundamental dynamics}

Insertion of the approximate control law (8) leads to a modified state space representation with new input $a$

$$
\dot{\boldsymbol{x}}_{\mathrm{p}}=\boldsymbol{f}_{\mathrm{p}}\left(\boldsymbol{x}_{\mathrm{p}}\right)+{ }^{a} \boldsymbol{g}_{\mathrm{p}}\left(\boldsymbol{x}_{\mathrm{p}}\right) a \text {. }
$$

In the following we show that through the transformation of the system input we obtain decoupled fundamental dynamics. Our assumptions for the fundamental system behavior are as follows:

- The phase $\varphi$ is approximately time-linear $\dot{\varphi} \approx \omega$ and the influence of the actuation $a$ on the phase is small.

- The energy flow $\dot{\vartheta}_{E} \approx \dot{\vartheta}_{r}$ is approximately equal to the amplitude of the actuation $a$ times a system dependent factor $B: \dot{\vartheta}_{r} \approx B a$ and thus zero for no actuation $a=0$.

In order to keep the error caused by the approximations $\vartheta_{E} \approx \vartheta_{r}$ and $\dot{\varphi} \approx \omega$ as small as possible, we use the actual natural frequency for normalization of the phase space $\Omega=\omega$ (see Fig.2).

We simplify each term of the state space representation in order to extract the fundamental dynamics through two consecutive steps:

S1 Approximations through 3rd order Taylor polynomials, e.g. $\sin \left(\vartheta_{r} \cos \varphi\right) \approx \vartheta_{r} \cos \varphi-\frac{\vartheta_{r}^{3} \cos ^{3} \varphi}{6}$

S2 Negligence of higher harmonics,

e.g. $\cos ^{4} \varphi=\frac{3}{8}+\frac{\cos 2 \varphi}{2}+\frac{\cos 4 \varphi}{8} \approx \frac{3}{8}$

1) Phase dynamics $\dot{\varphi}$ :

$$
\begin{aligned}
f_{\mathrm{p}, 1} & \stackrel{\Omega \equiv \omega}{=} \omega \sin ^{2} \varphi+\frac{\omega_{0}^{2}}{\omega \vartheta_{r}} \cos \varphi \sin \left(\vartheta_{r} \cos \varphi\right) \\
& \stackrel{\mathrm{S} 1, \mathrm{~S} 2}{\approx} \frac{1}{2} \omega+\frac{1}{2} \frac{\omega_{0}^{2}}{\omega}\left(1-\frac{1}{2}\left(\frac{\vartheta_{r}}{2}\right)^{2}\right) \\
& \stackrel{\mathrm{S} 1^{-1}}{\approx} \frac{1}{2} \omega+\frac{1}{2} \frac{\omega_{0}^{2}}{\omega}\left(\cos \left(\frac{\vartheta_{r}}{2}\right)\right) \\
& \stackrel{\omega_{g}(5)}{\approx} \omega,
\end{aligned}
$$

where " $\mathrm{S} 1^{-1}$ " indicates the reverse direction of a 3rd order Taylor approximation. Application of the approximation steps S1 and S2 to ${ }^{a} g_{\mathrm{p}, 1}$ lead to ${ }^{a} g_{\mathrm{p}, 1} \approx 0$. Consequently, insertion of the geometric mean approximation (5) in the last step in (21) results in the expected relationship $\dot{\varphi} \approx \omega$.

2) Energy dynamics $\dot{\vartheta}_{r}$ : Similar to ${ }^{a} g_{\mathrm{p}, 1}$, the approximation steps 1 and 2 result in $f_{\mathrm{p}, 2} \approx 0$. The remaining term ${ }^{a} g_{\mathrm{p}, 2}$ simplifies to

$$
\begin{aligned}
{ }^{a} g_{\varphi, 2} & =\frac{\omega \omega_{0}^{2}}{g} \sin ^{2} \varphi \cos \left(\vartheta_{r} \cos \varphi\right) \\
& \stackrel{\mathrm{S} 1, \mathrm{~S} 2}{\approx} \frac{1}{2 g} \omega \omega_{0}^{2}\left(1-\frac{1}{2}\left(\frac{\vartheta_{r}}{2}\right)^{2}\right) \\
& \stackrel{\mathrm{S}{ }^{-1}}{\approx} \frac{1}{2 g} \omega \omega_{0}^{2}\left(\cos \left(\frac{\vartheta_{r}}{2}\right)\right) \\
& \stackrel{\omega_{g}(5)}{\approx} \frac{1}{2 g} \omega^{3}=: B .
\end{aligned}
$$

Similar to (21), application of the inverse 3rd order Taylor approximation and the insertion of the geometric mean 


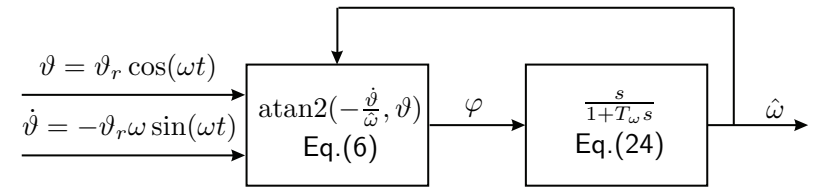

Fig. 3. Block diagram of the $\omega$-adaptation. Phase computation with normalization factor $\Omega=\hat{\omega}$.

approximation of the natural frequency $\omega$ of (5) lead to the expected energy dynamics $\dot{\vartheta}_{r} \approx B a$.

The decoupled fundamental simple pendulum dynamics result in

$$
\begin{aligned}
\dot{x}_{\mathrm{p}} & =\left(\begin{array}{c}
\dot{\varphi} \\
\dot{\vartheta}_{r}
\end{array}\right)=\left(\begin{array}{c}
\omega \\
B a
\end{array}\right) \\
\text { with } B & =\frac{1}{2 g} \omega^{3}=\text { const, }
\end{aligned}
$$

and are used in Section IV to design an adaptive energybased swing-up controller. Note that for the fundamental dynamics we make the assumption of a constant natural frequency $\omega$, and thus an harmonic oscillator. As the natural frequency is an unknown system parameter that will be adapted online, we are able to capture the nonlinear nature of the simple pendulum to a certain extent.

\section{AdAPTIVE SWING-UP CONTROL}

In this section we use the fundamental dynamics (23) derived in the previous section to design an adaptive swingup controller. The natural frequency $\omega$ is the only unknown system parameter and is adapted based on the phase $\varphi$. The system parameter $B$ follows from the fundamental dynamics with the natural frequency estimate $\hat{\omega}$. Based on $B$, we adapt a control gain $k$ such that the system follows the reference dynamics specified in the problem statement (13).

\section{A. Adaptation of natural frequency $\omega$}

According to the fundamental dynamics, the unknown system parameter $\omega$ equals the time derivative of the state $\varphi$. We capture this simple relationship in our adaptation dynamics, which additionally contain a first-order low-pass filter with cut-off frequency $\frac{1}{T_{\omega}}$

$$
\hat{\omega}=\frac{s}{1+T_{\omega} s} \varphi .
$$

The block diagram in Fig. 3 illustrates how the $\omega$ adaptation is integrated into the swing-up controller. The internal feedback of the natural frequency estimate $\hat{\omega}$ for the computation of the phase $\varphi$ requires a stability analysis.

We carry out the stability analysis of the $\omega$-adaptation based on the assumption that the fundamental dynamics are valid. This implies that the system input $\omega$ is constant (changes only slowly) and thus $\varphi(t)=\omega t+\varphi(t=0)$. Without loss of generality we assume $\varphi(t=0)=0$ in the following. Calculation of the phase $\varphi$ using the angle $\vartheta=\vartheta_{r} \cos (\omega t)$, angular velocity $\dot{\vartheta}=-\vartheta_{r} \omega \sin (\omega t)$ and normalization factor $\Omega=\hat{\omega}$ results in

$$
\varphi=\operatorname{atan} 2\left(-\frac{\dot{\vartheta}}{\hat{\omega}}, \vartheta\right)=\operatorname{atan} 2\left(-\frac{\omega}{\hat{\omega}} \sin (\omega t), \cos (\omega t)\right) \text {, }
$$

which is independent of the system energy $\vartheta_{r}$. Thus, the only input to the $\omega$-adaptation system in Fig. 3 is the natural frequency $\omega$ and the output is the estimate $\hat{\omega}$.

Transformation of the adaptation dynamics from frequency domain (24) to time domain results in

$$
\dot{\hat{\omega}}=-\frac{1}{T_{\omega}}(\hat{\omega}-\dot{\varphi}) .
$$

The time derivative of the phase $\varphi(25)$ is

$$
\dot{\varphi}=\frac{\omega\left(\tan (\omega t)\left(-\frac{1}{\hat{\omega}^{2}}\right) \dot{\hat{\omega}}+\frac{1+\tan ^{2}(\omega t)}{\hat{\omega}} \omega\right)}{1+\left(\frac{\omega}{\hat{\omega}} \tan (\omega t)\right)^{2}} .
$$

Insertion of (27) into (26) and some rearrangements result in the dynamics

$$
\dot{\hat{\omega}}=\frac{\hat{\omega} \omega^{2}-\hat{\omega}^{3}}{T_{\omega} \hat{\omega}^{2}+T_{\omega} \omega^{2} \tan ^{2}(\omega t)-\omega \tan (\omega t)},
$$

for which stability has to be shown. We choose the Lyapunov function

$$
V=\frac{1}{2}(\hat{\omega}-\omega)^{2}
$$

which leads through temporal differentiation to

$$
\dot{V}=\frac{-\hat{\omega}(\hat{\omega}-\omega)^{2}(\hat{\omega}+\omega)}{T_{\omega} \omega^{2} \tan ^{2}(\omega t)-\omega \tan (\omega t)+T_{\omega} \hat{\omega}^{2}} .
$$

The enumerator of $(30)$ is $-\hat{\omega}(\hat{\omega}-\omega)^{2}(\hat{\omega}+\omega) \leq 0$. The denominator is a quadratic function of $\tan (\omega t)$, where $\tan (\omega t)$ can take any values between $\pm \infty$. From the sign of the multiplier of the quadratic term $T_{\omega} \omega^{2}>0$ we know that the denominator with $\tan (\omega t)=x$ forms a parabola which opens up. Thus, a positive denominator is equivalent to a negative discriminant $D$

$$
D=\omega^{2}-4 T_{\omega} \omega^{2} T_{\omega} \hat{\omega}^{2}<0 \Rightarrow T_{\omega}>\frac{1}{2 \hat{\omega}} .
$$

This constraint implies that the adaptation speed is limited. Under the constraint (31), the time derivative of the Lyapunov function is strictly negative $\dot{V}<0$ subject to $\omega \neq \hat{\omega}$. This proves asymptotic stability of the $\omega$-adaptation under the assumption of the fundamental dynamics. For a linearly oscillating pendulum, the estimate $\hat{\omega}$ converges to the actual natural frequency $\omega$.

\section{B. Computation of control gain $k$}

We reformulate the linear mapping (9) in terms of the energy equivalent $\vartheta_{r}^{\mathrm{d}}-\vartheta_{r}$

$$
a=\left\{\begin{array}{cl}
\bar{a} \operatorname{sgn}\left(\vartheta_{r}^{\mathrm{d}}-\vartheta_{r}\right) & \text { if } k\left|\vartheta_{r}^{\mathrm{d}}-\vartheta_{r}\right| \geq \bar{a} \\
k\left(\vartheta_{r}^{\mathrm{d}}-\vartheta_{r}\right) & \text { else. }
\end{array}\right.
$$

The stability analysis of the proportional controller with gain $k$ in the following shows that under the fundamental 
dynamics assumption we reach the desired energy level $\vartheta_{r}^{\mathrm{d}}$. The Lyapunov function is

$$
V=\frac{1}{2}\left(\vartheta_{\mathrm{r}}-\vartheta_{\mathrm{r}}^{\mathrm{d}}\right)^{2}
$$

with time derivative

$$
\dot{V}= \begin{cases}-B \bar{a}\left|\vartheta_{r}^{\mathrm{d}}-\vartheta_{r}\right| & \text { if } k\left|\vartheta_{r}^{\mathrm{d}}-\vartheta_{r}\right| \geq \bar{a} \\ -B k\left(\vartheta_{r}^{\mathrm{d}}-\vartheta_{r}\right)^{2} & \text { else. }\end{cases}
$$

Consequently, $\dot{V}<0$ subject to $\vartheta_{r} \neq \vartheta_{r}^{\mathrm{d}}$ and for all $k, B, \bar{a}>0$ the energy level $\vartheta_{r}=\vartheta_{r}^{\mathrm{d}}$ resembles a globally asymptotically stable fixpoint ${ }^{2}$.

As stated in the problem formulation (13), the swing-up process is supposed to not only reach the desired energy level $\vartheta_{r}^{\mathrm{d}}$, but to behave as the first-order reference dynamics

$$
\dot{\vartheta}_{r \mathrm{~m}}=K_{\mathrm{d}}\left(\vartheta_{r}^{\mathrm{d}}-\vartheta_{r \mathrm{~m}}\right),
$$

formulated here in terms of the phase space radius $\vartheta_{r}$. Off the saturation, we obtain the proportional controller $a=k\left(\vartheta_{\mathrm{r}}^{\mathrm{d}}-\vartheta_{\mathrm{r}}\right)$. Insertion of the fundamental dynamics (23) yields

$$
\dot{\vartheta}_{r}=B k\left(\vartheta_{r}^{\mathrm{d}}-\vartheta_{r}\right) .
$$

Comparison of (35) and (36) leads to the requirement $k B \stackrel{!}{=} K_{\mathrm{d}}$ and thus the control gain

$$
k=\frac{K_{\mathrm{d}}}{B} \stackrel{(23)}{=} \frac{2 g K_{\mathrm{d}}}{\omega^{3}} .
$$

Saturation of the controller means that tracking of the first order reference dynamics requires an amplitude $a$ that violates the amplitude limits $\pm \bar{a}$.

\section{SiMULATION EXPERIMENT}

The simple form of the fundamental dynamics derived in Section III allows for the design of a stable adaptive controller in Section IV. However, the fundamental dynamics only approximate the behavior of a simple pendulum. In this section, three simulation experiments are performed with the purpose to analyze the controller behavior in interaction with a simple pendulum:

1) Analysis of the $\omega$-adaptation

2) Analysis of the follow-up behavior with respect to the reference dynamics

3) Analysis of the complete controller under more realistic circumstances and stabilization in the unstable equilibrium

\section{A. Simulation setup}

The simulation experiments are performed using MAT$L A B /$ Simulink. The first two simulation experiments use the undamped simple pendulum (1). For the third simulation experiment we introduce viscous damping of $d=-\frac{\partial \ddot{\vartheta}}{\partial \dot{\vartheta}}=0.11 / \mathrm{s}$. The mass of the simple pendulum is $m=0.5 \mathrm{~kg}$. We choose the length such that the small angle approximation of the natural frequency is

\footnotetext{
${ }^{2}$ In reality $B \geq 0$. Asymptotic stability can still be shown for all initial states except $\vartheta_{r}=0$ using e.g. LaSalle's Invariance Principle [15].
}

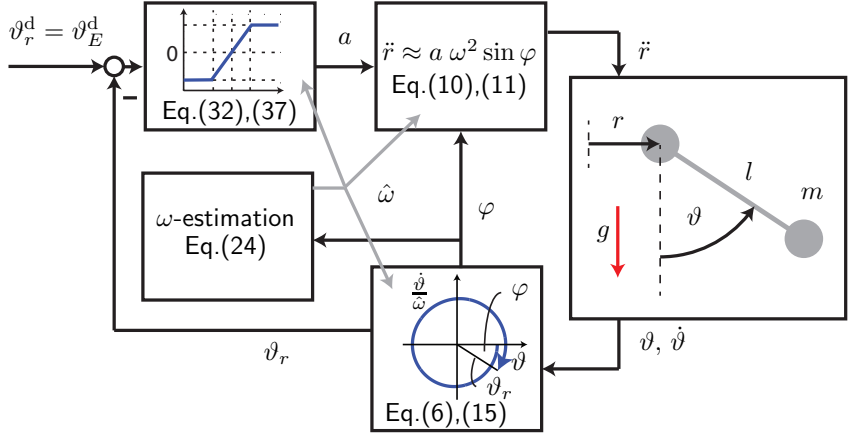

Fig. 4. Block diagram of the simulated system. For equations with normalization factor $\Omega=\hat{\omega}$.

$\omega_{0}=\sqrt{\frac{g}{l}}=4 \mathrm{rad} / \mathrm{s}$. The initial pendulum configuration is $\vartheta(t=0)=2^{\circ}, \dot{\vartheta}(t=0)=0$. The adaptive controller as well as the original controller by [10] cannot start from the lower equilibrium point. For $\vartheta(t=0)=\dot{\vartheta}(t=0)=0$, the phase $\varphi$ stays zero with the effect of zero acceleration $\ddot{r}$. In experiments this problem can be circumvented, e.g. through a controlled initial jerk in $\ddot{r}$.

The block diagram in Fig. 4 shows the entire system. The natural frequency estimate $\hat{\omega}$ is input to the transformation from cartesian $\boldsymbol{x}_{\mathrm{c}}$ to polar states $\boldsymbol{x}_{\mathrm{p}}$, for the computation of the fundamental dynamics constant $B$ as well as for the filter design and the reference trajectory $r^{\mathrm{d}}$. The controller parameters that stay fixed for all simulation experiments are $K_{\mathrm{d}}=0.4^{1} / \mathrm{s}, c_{\mathrm{f}}=0.9$ and $\zeta=1.2$.

Within the last simulation experiment, we show that the presented adaptive controller can be used to inject energy into the pendulum such that it reaches its unstable equilibrium. The adaptive energy-based controller turns the upper equilibrium point into an unstable saddle point [4]. Consequently, we need to switch to a linear controller to stabilize the inverted pendulum. We apply an LQR controller based on the linearized state space representation (1) around the unstable equilibrium point $x_{\mathrm{c}}^{*}=\left(\begin{array}{ll}\pi & 0\end{array}\right)$, with $Q=\left(\begin{array}{cc}0.09 & 0 \\ 0 & 0.09\end{array}\right)$ and $R=1$. Note, as the focus of our work is on the adaptive swing-up controller, we assume complete system knowledge for the design of the stabilizing controller. We switch from the swing-up controller to the LQR controller if $\left|2 m g l-E_{\vartheta}\right|<0.05 \mathrm{~J}$ or $1-\cos (\vartheta-\pi)<0.09$ as in [10].

\section{B. Simulation results and discussion}

1) Analysis of w-adaptation: Figure 5 shows the simulation results for three different filter time constants $T_{\omega}$ of the $\omega$-adaptation (24). We obtain accurate values for the natural frequency $\omega=\omega_{\mathrm{M} 4}$ from the 4th iteration of the arithmetic-geometric mean computation [14]. The initial value of the natural frequency estimate is $\hat{\omega}(t=0)=1 \mathrm{rad} / \mathrm{s}$. The lossless simple pendulum is actuated with a constant amplitude factor $a=0.04 \mathrm{~m}$. Despite the constant amplitude, the pendulum swing-up is slightly affected by the adaptation process, as the estimated natural frequency defines the cut-off frequency of the second-order low-pass filter (11). 


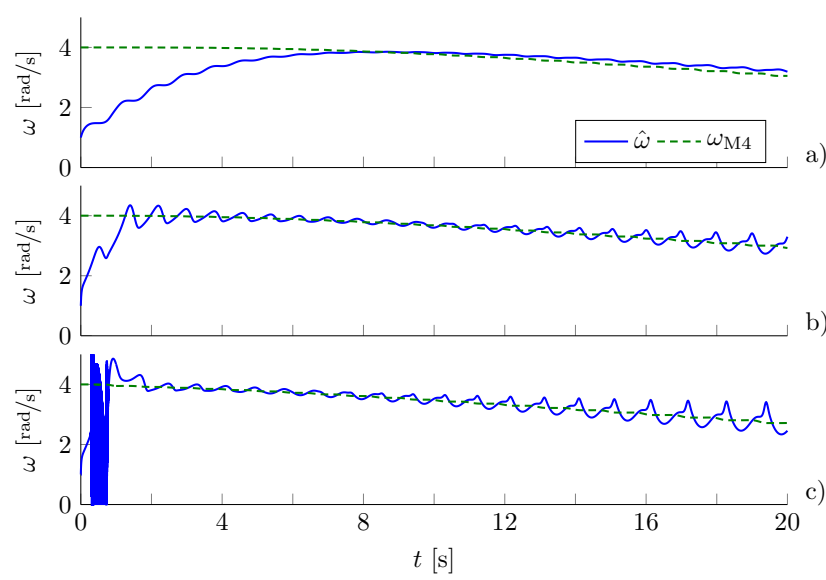

Fig. 5. Analysis of $\omega$-adaptation: Natural frequency $\omega$ and its estimate $\hat{\omega}$ for a) $T_{\omega}=2 \mathrm{~s}$, b) $T_{\omega}=0.2 \mathrm{~s}$ and c) $T_{\omega}=0.17 \mathrm{~s}$ of a lossless simple pendulum actuated with constant amplitude $a=0.04 \mathrm{~m}$.

The constant actuation increases the system energy over time with the effect of a decreasing natural frequency $\omega$ (see Fig. 5). We observe adaptation to the decreasing natural frequency $\omega$ for all three time constants $T_{\omega}$. The adaptation process is faster for lower time constants $T_{\omega}$, with instabilities visible for $T_{\omega}=0.17 \mathrm{~s}$. Lower time constants $T_{\omega}$ and higher energy levels lead to increased oscillations of the estimate $\hat{\omega}$ around the actual natural frequency.

Discussion: The high frequent oscillations for the lowest time constant $T_{\omega}$ strengthen our result in Section IVA that the adaptation speed is limited. According to the stability analysis, the theoretical constraint is $T_{\omega}>\frac{1}{2 \hat{\omega}}$. The natural frequency estimate during the simulation ranges from $\hat{\omega}=[1,5] \mathrm{rad} / \mathrm{s}$. Consequently, we expect stable system behavior for time constants $T_{\omega}>0.5 \mathrm{~s}$. Note that the constraint found based on Lyapunov theory might be conservative, also for the dynamics (28) found based on the fundamental dynamics. Simulations with the simple pendulum show first small regions of unstable behavior for $T_{\omega} \leq 0.17 \mathrm{~s}$. The fact that the stability bound found in simulation is close to the theoretically found stability bound, supports the fundamental dynamics approximation. The oscillations around the actual value of the natural frequency $\omega$ show the limitations of the fundamental dynamics approximation. With increasing energy content the nonlinearities of the simple pendulum become more apparent and the fundamental dynamics approximation less accurate. Lower time constants $T_{\omega}$ attenuate the erroneous oscillations in $\hat{\omega}$. Thus, the time constant $T_{\omega}$ can be used to find a compromise between fast adaptation and sufficient filtering.

2) Analysis of follow-up behavior: The second simulation experiment investigates how well the reference dynamics $\vartheta_{r \mathrm{~m}}$ are followed by the system energy $\vartheta_{E}$. For this purpose, we replace the $\omega$-adaptation with the accurate results of the arithmetic-geometric mean computation $\omega_{\mathrm{M} 4}$. We set the desired energy to $\vartheta_{E}^{\mathrm{d}}=120^{\circ}$. Figure 6 shows the simulation results for two different amplitude limits $\bar{a}=0.1 \mathrm{~m}$ and $\bar{a}=1 \mathrm{~m}$. For the higher limit $\bar{a}$, the amplitude $a$ does not
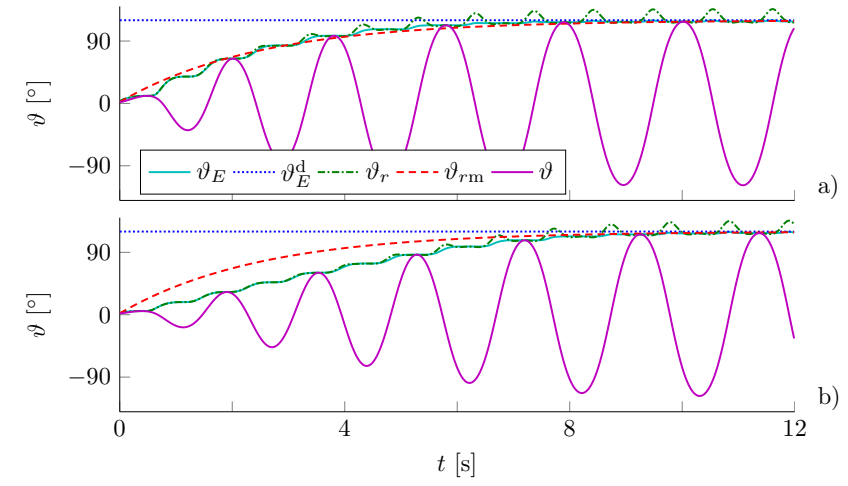

Fig. 6. Analysis of follow-up behavior: Angle $\vartheta$, desired energy $\vartheta_{E}^{\mathrm{d}}$, reference dynamics $\vartheta_{r \mathrm{~m}}$ and energy equivalents $\vartheta_{E}$ and $\vartheta_{r}$ of a lossless simple pendulum with accurate $\omega$ estimates for amplitude limits a) $\bar{a}=1 \mathrm{~m}$ and b) $\bar{a}=0.1 \mathrm{~m}$.

saturate $(a<0.25 \mathrm{~m})$ with the effect that the energy equivalents $\vartheta_{E}$ and $\vartheta_{r}$ closely track the reference $\vartheta_{r \mathrm{~m}}$. Saturation of the amplitude $a$, as it occurs for the lower limit $\bar{a}$, prohibits a swing-up equally fast as the reference $\vartheta_{r m}$. During the swing-up, the phase space radius $\vartheta_{r}$ is almost equal to the accurate energy equivalent $\vartheta_{E}$. While the accurate energy equivalent $\vartheta_{E}$ shows that the system energy converges to the desired energy level $\vartheta_{E}^{\mathrm{d}}$, we observe oscillations of the energy approximation $\vartheta_{r}$ during the energetic steady state.

Discussion: The close tracking of the reference dynamics $\vartheta_{r \mathrm{~m}}$ for an unrestricted amplitude $a$ and a lossless simple pendulum with accurate $\omega$ estimates shows that the energy dynamics are well approximated by the fundamental dynamics $\dot{\vartheta}_{r}=B a$. The oscillations of the phase space radius $\vartheta_{r}$ expose the nonlinearities of the simple pendulum, which are more apparent for higher energy contents and are not perfectly captured by the fundamental dynamics. During swing-up, the phase portrait of the simple pendulum is more circle-like with the result of $\vartheta_{r} \approx \vartheta_{E}$ also for higher energies than shown in Fig. 6. During steady state, the phase portrait is less circle-like which becomes visible in higher deviations of $\vartheta_{r}$ from $\vartheta_{E}$. Figure 6 shows that despite of the inaccuracies of $\vartheta_{r}$ during the steady state, the system energy $\vartheta_{E}$ closely tracks the desired energy level $\vartheta_{E}^{\mathrm{d}}$.

3) Inverted pendulum experiment: For the third simulation experiment, we choose a more realistic setting. Instead of assuming full state-feedback, only noisy angle measurements $\vartheta_{\mathrm{n}}$ are available. A normally distributed random signal of frequency $1 \mathrm{kHz}$ with variance $5^{\circ}$ and zero mean serves as the noise. We filter the noisy angle measurements $\vartheta_{\text {n }}$ with a 8th-order low-pass butterworth filter with cut-off frequency $50 \mathrm{rad} / \mathrm{s}$. Numerical differentiation of the filtered angle measurements $\vartheta_{\mathrm{nF}}$ lead to estimates of the angular velocity $\dot{\vartheta}_{\mathrm{nF}}$. We set the desired energy level to the energy of the unstable equilibrium $\vartheta_{E}^{\mathrm{d}}=\pi$. The $\omega$-estimation starts from an initial value of $\hat{\omega}(t=0)=7 \mathrm{rad} / \mathrm{s}$ with time constant $T_{\omega}=0.5 \mathrm{~s}$. The amplitude factor is limited by $\bar{a}=0.2 \mathrm{~m}$.

Figure 7 shows the swing-up to and the stabilization at the unstable equilibrium. The simple pendulum follows 

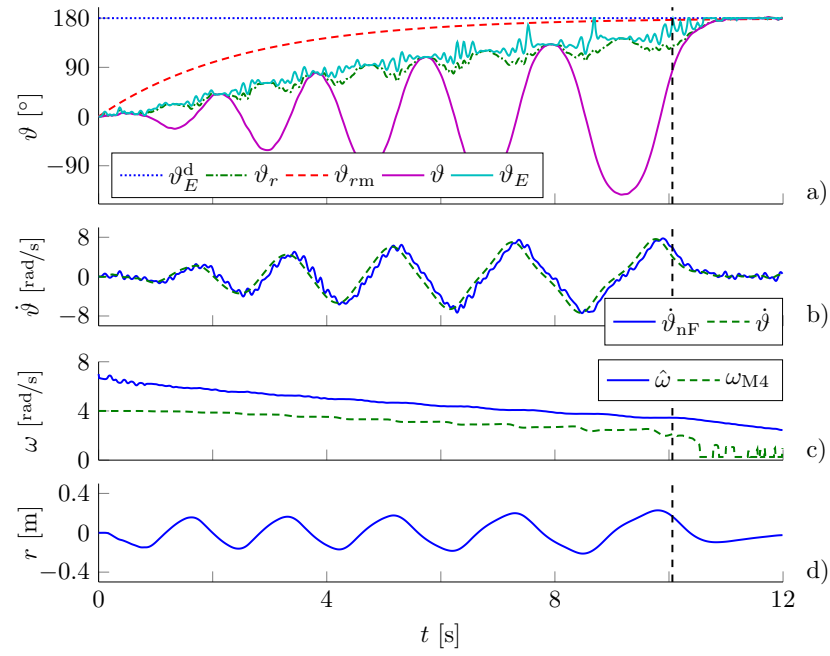

Fig. 7. Swing-up and stabilization of pendulum in its unstable equilibrium for a damped simple pendulum: a) Angle $\vartheta$, desired energy $\vartheta_{E}^{\mathrm{d}}$, reference dynamics $\vartheta_{r \mathrm{~m}}$, energy equivalents $\vartheta_{E}$ and $\vartheta_{r}$, b) angular velocity $\dot{\vartheta}$ and noise affected angular velocity $\dot{\vartheta}_{\mathrm{nF}}, \mathrm{c}$ ) natural frequency $\omega$ and its estimate $\hat{\omega}, \mathrm{d})$ trajectory $r$.

the reference dynamics with a clear delay, but reaches the region of the stabilizing LQR controller slightly after $10 \mathrm{~s}$ (dashed vertical line in Fig. 7). The noise added to the angle measurements with the subsequent filtering and numerical time differentiation leads to a disturbed angular velocity $\dot{\vartheta}_{\mathrm{nF}}$, subject to a clear phase shift with respect to the true angular velocity $\dot{\vartheta}$. The disturbed states $\vartheta_{\mathrm{nF}}$ and $\dot{\vartheta}_{\mathrm{nF}}$ affect the energy approximations $\vartheta_{E}$ and $\vartheta_{r}$. The natural frequency estimate $\hat{\omega}$ smoothly approaches but does not reach the actual natural frequency $\omega$. The amplitude $a$ stays within the limits $\bar{a}$ except for short periods of time around $9 \mathrm{~s}$ (not displayed) and the trajectory $r$ does not exceed $\pm 0.23 \mathrm{~m}$.

Discussion: The simulation demonstrates successful swing-up to the unstable equilibrium, close to which an LQR controller stabilizes the pendulum. The noise added to the angle measurement $\vartheta$ clearly affects the energy estimates. Furthermore, we initialized the $\omega$-estimation far away from its actual initial value. The initialization of the $\omega$-adaptation as well as the disturbed states $\vartheta_{r}$ and $\varphi$ cause the delay with respect to the reference dynamics $\vartheta_{r \mathrm{~m}}$. Nevertheless, the adaptive controller successfully injects energy into the pendulum, such that the pendulum reaches high energy levels and consequently the highly nonlinear regime. The performance of the adaptive controller in this more realistic setting supports the applicability of the fundamental dynamics approximation.

\section{CONCLUSION}

In this work, we design an adaptive energy-based swingup controller. A state transformation allows us to approximate the nonlinear simple pendulum dynamics in a simple decoupled fashion, the fundamental dynamics. Based on the fundamental dynamics, the natural frequency is adapted and reference dynamics are tracked. Convergence to the natural frequency and to the desired energy level are shown under the fundamental dynamics assumption. The interaction of the adaptive controller with a simple pendulum is investigated through simulation experiments. We verify the theoretically obtained stability bounds in simulation. Good tracking performance of the desired reference dynamics further supports the fundamental dynamics approximation. A controlled swing-up to the unstable equilibrium point based on noisy angle measurements indicates the applicability of the adaptive controller to real-world inverted pendulum experiments. In future work we plan to apply the approach to the manipulation of unknown complex pendulum-like objects.

\section{ACKNOWLEDGMENT}

The authors would like to thank Stefan Kersting and Markus Schill for their valuable feedback.

\section{REFERENCES}

[1] S. Kajita, F. Kanehiro, K. Kaneko, K. Yokoi, and H. Hirukawa, "The 3d linear inverted pendulum mode: a simple modeling for a biped walking pattern generation," in Proceedings IEEE/RSJ IROS, vol. 1, 2001, pp. 239-246 vol.1.

[2] J. Nakanishi, T. Fukuda, and D. Koditschek, "A brachiating robot controller," IEEE Transactions on Robotics and Automation, vol. 16 , no. 2, pp. 109-123, Apr 2000.

[3] P. Donner, F. Christange, and M. Buss, "Human-robot cooperative swinging of complex pendulum-like objects," in Proc. IEEE/RSJ IROS, Nov 2013, pp. 4602-4608.

[4] K. Åström and K. Furuta, "Swinging up a pendulum by energy control," Automatica, vol. 36, no. 2, pp. 287 - 295, 2000.

[5] M. Spong, "The swing up control problem for the acrobot," IEEE Control Systems, vol. 15, no. 1, pp. 49-55, Feb 1995.

[6] M. Spong and D. Block, "The pendubot: a mechatronic system for control research and education," in Proceedings IEEE CDC, vol. 1, Dec 1995, pp. 555-556 vol.1.

[7] K. Furuta, "Control of pendulum: from super mechano-system to human adaptive mechatronics," in Proceedings IEEE CDC, vol. 2, Dec 2003, pp. 1498-1507 Vol.2.

[8] C. C. Chung and J. Hauser, "Nonlinear control of a swinging pendulum," Automatica, vol. 31, no. 6, pp. 851 - 862, 1995.

[9] R. Lozano, I. Fantoni, and D. Block, "Stabilization of the inverted pendulum around its homoclinic orbit," Systems and Control Letters, vol. 40, no. 3, pp. 197-204, 2000.

[10] K. Yoshida, "Swing-up control of an inverted pendulum by energybased methods," in Proc. ACC, vol. 6, 1999, pp. 4045-4047.

[11] N. Zoso and C. Gosselin, "Point-to-point motion planning of a parallel 3-dof underactuated cable-suspended robot," in Proc. IEEE ICRA, May 2012, pp. 2325-2330.

[12] C. Tao, J. Taur, C. Wang, and U. Chen, "Fuzzy hierarchical swing-up and sliding position controller for the inverted pendulumcart system," Fuzzy Sets and Systems, vol. 159, no. 20, pp. 2763 - 2784, 2008.

[13] I. Palunko, P. Donner, M. Buss, and S. Hirche, "Cooperative suspended object manipulation using reinforcement learning and energy-based control," in Proc. IEEE/RSJ IROS, Sept 2014, pp. 885-891.

[14] C. G. Carvalhaes and P. Suppes, "Approximations for the period of the simple pendulum based on the arithmetic-geometric mean," American Journal of Physics, vol. 76, no. 12, pp. 1150-1154, 2008.

[15] J.-J. E. Slotine, W. Li et al., Applied nonlinear control. Prentice hall New Jersey, 1991, vol. 199, no. 1. 